\title{
Gestão terapêutica dos episódios de dor aguda autolimitada em utentes de um Centro de Saúde
}

Sílvia Batista, ${ }^{*}$ Inês Cordeiro, ${ }^{*}$ Nuno Florêncio, ${ }^{*}$ Duarte Rebelo, ${ }^{*}$ Flávio Simões, ${ }^{*}$ Maria Inês Taborda, ${ }^{*}$ Joana Teixeira*

\section{RESUMO}

Introdução: O tratamento da dor é uma competência do médico mas também dos indivíduos. A automedicação informada é benéfica para a população e para o Sistema de Saúde, mas incorrecções são frequentes, aumentando o risco de efeitos adversos. Objectivos: Caracterizar, na amostra em estudo, os episódios autolimitados de dor aguda e a gestão terapêutica dos mesmos. Estudar os casos de automedicação em termos de frequência e relação com factores sócio-demográficos.

Métodos: O estudo transversal foi realizado usando uma amostra de 352 utentes do Centro de Saúde do Cacém (extensão Olival) com recurso a questionário por entrevista pessoal acerca de dor aguda autolimitada nos 30 dias anteriores e sua gestão terapêutica. Resultados: contabilizaram-se 89 episódios de dor aguda autolimitada (25,3\%), maioritariamente mulheres. Os episódios mais frequentes foram cefaleias (48,3\%), mialgias e cefaleias relacionadas com síndrome gripal (10,1\%) e raquialgias (7,9\%). Dos 89 utentes que referiram ter tido um episódio de dor agudo autolimitado nos 30 dias antecedentes ao questionário, $63,0 \%$ recorreram unicamente a terapêutica farmacológica, com 12,4\% recorrendo também a terapêutica não farmacológica. Paracetamol $(50,7 \%)$ e AINEs $(31,3 \%)$ foram os fármacos mais utilizados. A automedicação foi muito frequente $(46,5 \%)$ e ocorreu principalmente em mulheres e em idades inferiores a 45 anos, associando-se a doses sub-terapêuticas, com base igualmente em indicação médica anterior ou em auto-decisão.

Discussão: Verificou-se que há associações significativas entre a automedicação e alguns factores sócio demográficos e que aquela interfere com a posologia adequada. Também se observou que a autodecisão é muito importante dentro da automedicação, sugerindo-se que os utentes devam ser mais bem esclarecidos sobre as propriedades dos fármacos.

Palavras-Chave: Epidemiologia da dor; Frequência; Dor Aguda; Automedicação.

\section{INTRODUÇÃO}

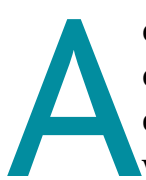
dor define-se como uma sensação subjectiva de desconforto físico, ${ }^{1}$ sendo por isso um obstáculo à saúde. É um dos principais motivos de consulta médica, nomeadamente no âmbito da Medicina Geral e Familiar. A sua gestão e tratamento têm custos muito elevados. Nos Estados Unidos, calcula-se que sejam gastos anualmente 100 mil milhões de dólares no seu tratamento, ${ }^{2}$ dos quais 62 mil milhões de dólares se devem exclusivamente à diminuição da produtividade das pessoas afectadas. ${ }^{3}$ Infere-se então

*Faculdade de Medicina da Universidade de Lisboa que a dor tem um impacto profundo na saúde individual, no funcionamento da sociedade e na economia.

Dada a elevada frequência de episódios dolorosos ao longo da vida de um indivíduo, a gestão da dor é também uma competência do próprio e não unicamente do médico. A automedicação consciente e informada é benéfica para a população, tornando-a mais participativa e responsável pela sua saúde, o que diminui o recurso a consultas, aliviando a pressão sobre o Sistema de Saúde e reduzindo os gastos estatais. ${ }^{4}$ Contudo, os gastos transitam para a população, que não beneficia de descontos sociais na compra de fármacos de venda livre. $^{5}$ 
Vários estudos internacionais indicam que os indivíduos baseiam frequentemente a sua automedicação em informações incorrectas: ${ }^{6-8}$ uma elevada percentagem da população considera os fármacos mais dispendiosos mais eficientes, desconhece os seus efeitos secundários, não respeita as doses terapêuticas e utiliza os fármacos fora das suas indicações. A automedicação com base em pessoas não qualificadas revelou-se tão frequente como a com base em directrizes credíveis. Este facto, aliado ao desconhecimento por parte dos médicos das práticas de automedicação dos seus doentes, aumenta francamente o risco populacional de efeitos secundários e adversos. ${ }^{?}$

Em Portugal, são escassos os estudos publicados a respeito da automedicação, mas estima-se que o interesse pelo tema tenderá a crescer com o advento dos fármacos de venda livre.

\section{OBJECTIVOS}

Este estudo teve os seguintes objectivos: 1) Caracterizar os episódios de dor aguda e relacioná-los com variáveis demográficas; 2) Determinar a frequência de automedicação e relacioná-la com factores sócio-demográficos e da dor; 3) Determinar a frequência de erros na automedicação e gestão da dor; 4) Estudar qual o tipo de informação em que os utentes baseiam as suas estratégias terapêuticas; 5) Avaliar a confiança nas informações fornecidas por vários veículos de informação para a saúde.

\section{FINALIDADE}

Contribuir para a caracterização dos episódios de dor aguda e da gestão terapêutica dos mesmos, no âmbito dos Cuidados de Saúde Primários.

\section{MATERIAL E MÉTODOS}

Realizou-se um estudo transversal e analítico com utentes que recorreram ao Centro de Saúde (CS) do Cacém, extensão Olival, com idade superior a 15 anos, durante uma manhã e duas tardes em dois dias do mês de Janeiro de 2007. No inquérito anónimo foram recolhidos os dados referentes às características do indivíduo e à gestão terapêutica de episódios álgicos agudos resolvidos, decorridos durante os 30 dias anteriores ao estudo. Foram excluídas deste estudo as grávidas, pela sua maior ligação ao SNS e o uso quase exclusivo do paracetamol.
Do inquérito realizado constavam alguns itens, como: 1) se teve algum episódio de dor aguda autolimitada (definida ao entrevistado como dor de início e término nos 30 dias anteriores à data de entrevista, não relacionada com agudização de patologia crónica e controlada com algum tipo de terapêutica sintomática); 2) idade em anos (posteriormente recodificada em grupos etários); 3) sexo; 4) origem étnica. Aos entrevistados que apresentaram episódios agudos resolvidos foi ainda questionada: 5) escolaridade; 6) medicação habitual (número total de fármacos diferentes); 7) localização, intensidade (perante uma escala de graduação que considerava dor sem prejuízo das actividades diárias, com prejuízo ligeiro, moderado ou grave) e duração dos episódios álgicos; 8) se efectuou terapêutica não farmacológica e qual; 9) medicação utilizada - princípio activo e dose; ${ }^{9} 10$ ) qual o tempo entre o início da dor e início da toma da medicação, em horas ou dias; 11) quem recomendou o(s) fármaco(s); 12) se o fármaco foi tomado em regime de automedicação, qual a base para decisão do fármaco e doses utilizadas; 13) quanto tempo durou a terapêutica, em horas ou dias; 14) se houve remissão da dor; 15) grau de confiança do doente em relação ao médico, farmacêutico/enfermeiro, medicinas alternativas, sabedoria popular e comunicação social. A utilização de perguntas de resposta aberta deve-se à necessidade de conhecimentos da área da saúde para responder às questões. A opção pelo método de entrevista tornou possível a inclusão de pessoas com baixa escolaridade.

Fez-se um estudo estatístico sequencial dos dados recolhidos utilizando o software SPSS v.14 para Windows: 1) descritivo da amostra; 2) descritivo das características dos utentes com episódio de dor aguda; 3) analítico do possível estabelecimento de associações através do teste de qui-quadrado de acordo com os objectivos estabelecidos. Verificou-se a impossibilidade de tratar estatisticamente os dados referentes a quatro utentes, que apresentaram mais do que um episódio de dor aguda resolvida, pelo que se decidiu excluí-los do estudo.

A exclusão de um período matinal diminuiu a representação na amostra dos utentes que frequentam o Centro de Saúde nesse horário, eventualmente com características diferentes dos frequentadores do período da tarde. A exclusão dos restantes dias da semana tam- 
bém pode ter incluído maior representação relativa de grupos particulares de utentes e deixado fora do estudo outros grupos.

\section{RESULTADOS}

A amostra foi constituída por 352 utentes do Centro de Saúde do Cacém (extensão do Olival), com idades compreendidas entre os 16 e os 83 anos, dos quais 114 do sexo masculino e 238 do sexo feminino, que recorreram ao Centro de Saúde nos dois dias durante os quais o inquérito foi efectuado. A idade média era 46,64 \pm 17,11 anos, com 307 indivíduos de raça caucasiana e 45 de raça negra.

Dos inquiridos, $263(74,7 \%)$ não tiveram nenhum episódio de dor aguda resolvida nos últimos 30 dias, enquanto 89 tiveram um episódio de dor aguda resolvida nos últimos 30 dias (25,3\%). As características destes últimos estão descritas no Quadro I.

Os tipos de episódios álgicos foram cefaleias (48,3\%), mialgias e cefaleias relacionadas com síndrome gripal $(10,1 \%)$, raquialgia $(7,9 \%)$, dor abdominal $(6,7 \%)$, dor torácica $(5,6 \%)$, odinofagia $(6,7 \%)$, dismenorreia $(4,5 \%)$, dor de dentes $(3,4 \%)$, dor osteo-articular $(3,4 \%)$, dor traumática $(2,2 \%)$, dor por lesão cutânea $(2,2 \%)$. Relativamente à intensidade da dor em relação à realização das actividades diárias: $32,6 \%$ tiveram dor sem prejuízo das mesmas, $21,4 \%$ tiveram prejuízo ligeiro, 20,2\% tiveram prejuízo moderado e $25,8 \%$ tiveram prejuízo grave. Em termos de duração da dor, esta durou até uma hora em 11,2\%; entre uma e seis horas em 19,1\%; entre seis e 24 horas em 19,1\%; entre um e três dias em 23,6\%; entre quatro e sete dias em 16,9\% e mais de uma semana em $10,1 \%$ dos utentes.

Dos 89 utentes que apresentaram um episódio álgico, verificou-se que $63,0 \%$ recorreram unicamente a terapêutica farmacológica, 8,0\% recorreram apenas a terapêutica de alívio não farmacológico, 12,0\% recorreram a ambas e 17,0\% não recorreram a qualquer tipo de terapêutica.

Na terapêutica não farmacológica, 50,0\% tomou chás, $16,6 \%$ fez repouso, $11,0 \%$ adoptou posição antálgica, 5,6\% realizou massagem, 5,6\% tomou banho quente, 5,6\% aplicou um emplastro "natural" e 5,6\% esteve em privação sensorial.

Dos utentes que realizaram qualquer tipo de terapêutica farmacológica $(n=67), 50,7 \%$ utilizaram paracetamol, 31,3\% utilizaram AINE, 4,5\% utilizaram associação antigripal, 3,0\% utilizaram associação antigripal e AINE, 3,0\% utilizaram anti-inflamatório tópico, $3,0 \%$ utilizaram antibiótico, $1,5 \%$ utilizaram dois anti-inflamatórios não-esteróides (AINE), 1,5\% utilizaram associação de paracetamol com ergotamina e 1,5\% utilizaram paracetamol com relaxante muscular. A terapêutica foi utilizada em dose subterapêutica em $29,2 \%$ dos casos, em dose terapêutica em $53,8 \%$, em dose supraterapêutica em 10,8\% e em dose não especificada em $5,2 \%$ dos casos.

O início da terapêutica ocorreu de imediato em $22,9 \%$ dos casos, até uma hora em $21,4 \%$ dos casos, entre uma e seis horas em $24,3 \%$ dos casos, até 12 horas em 5,7\% dos casos, até 24 horas em 10,0\% dos casos, mais de 24 horas em $15,7 \%$ dos casos. A terapêutica foi

\section{QUADRO I. Características dos utentes com episódio de dor aguda resolvido $(\mathrm{n}=\mathbf{8 9})$}

\begin{tabular}{|c|c|c|c|c|c|}
\hline Características & Categorias & Frequência (\%) & Características & Categorias & Frequência (\%) \\
\hline \multirow{2}{*}{ Sexo } & Masculino & $23(25,8)$ & \multirow{7}{*}{ Grupo etário } & $16-25$ & $12(13,5)$ \\
\hline & Feminino & $66(74,2)$ & & $26-35$ & $20(22,5)$ \\
\hline \multirow{2}{*}{ Origem étnica } & Caucasiana & $74(83,1)$ & & $36-45$ & $16(18,0)$ \\
\hline & Negra & $15(16,9)$ & & $46-55$ & $9(10,0)$ \\
\hline \multirow{6}{*}{ Escolaridade } & Analfabetos & $3(3,4)$ & & $56-65$ & $16(18,0)$ \\
\hline & $1^{\circ}$ Ciclo / $4^{a}$ Classe & $31(34,8)$ & & $66-75$ & $12(13,5)$ \\
\hline & $2^{\circ}$ Ciclo $/ 6^{\circ}$ Ano & $5(5,6)$ & & $76-85$ & $4(4,5)$ \\
\hline & $3^{\circ} \mathrm{Ciclo} / 9^{\circ}$ Ano & $21(23,6)$ & \multirow{3}{*}{$\begin{array}{l}\text { Medicação } \\
\text { habitual }\end{array}$} & 0 fármacos & $33(37,1)$ \\
\hline & $12^{\circ}$ Ano & $20(22,5)$ & & 1-2 fármacos & $37(41,6)$ \\
\hline & Curso Profissional & $2(2,2)$ & & 3-4 fármacos & $10(11,2)$ \\
\hline
\end{tabular}




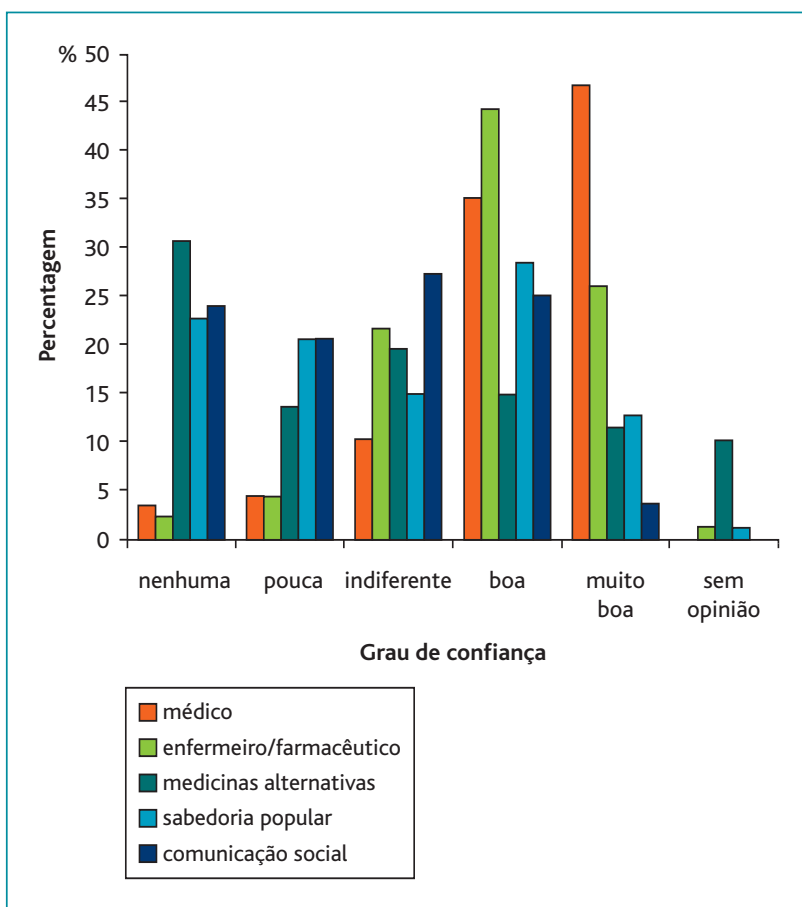

Figura 1. Grau de confiança dos utentes com episódios de dor aguda resolvida.

recomendada por médicos em $29,6 \%$ dos casos, por farmacêuticos/enfermeiros em $14,1 \%$, por conhecidos em $9,8 \%$ e pelo próprio (automedicação) em $46,5 \%$. Nos casos de automedicação, os utentes utilizaram-na em $41,7 \%$ baseando-se em prescrições médicas anteriores; $47,2 \%$ por iniciativa própria; $2,8 \%$ por recomendação anterior do farmacêutico; $2,8 \%$ por sugestão de conhecidos e $5,5 \%$ por outras razões. Dos inquiridos que tiveram episódio de dor aguda resolvida nos últimos 30 dias e que realizaram terapêutica farmacológica, $11,2 \%$ refere que o episódio doloroso não resolveu e cerca de $88,8 \%$ refere que cessou.

A Figura 1 indica a distribuição do grau de confiança dos utentes acerca das fontes de informação para a saúde.

Verificou-se que nos utentes do sexo feminino é mais frequente a prática de automedicação que nos utentes do sexo masculino (Quadro II). Nos utentes com 45 anos ou menos medicados farmacologicamente, predomina a automedicação, enquanto que nos com mais de 45 anos predomina a não automedicação (Quadro III).

\begin{tabular}{|c|c|c|c|c|}
\hline \multicolumn{5}{|c|}{$\begin{array}{l}\text { QUADRO II. Análise da associação entre a } \\
\text { automedicação e o sexo }(p<0,05)\end{array}$} \\
\hline \multirow[b]{2}{*}{ Sexo (\%) } & \multicolumn{2}{|c|}{ Automedicação } & \multirow{2}{*}{$\begin{array}{l}\text { Total } \\
(\%)\end{array}$} & \multirow{2}{*}{$\begin{array}{c}\text { Teste } \\
\text { (pearson } \\
\text { qui-quadrado) }\end{array}$} \\
\hline & Sim & Não & & \\
\hline Feminino & 59,6 & 40,4 & 100 & \multirow{2}{*}{$p=0,003$} \\
\hline Masculino & 14,3 & 85,7 & 100 & \\
\hline
\end{tabular}

QUADRO III. Análise da associação entre a

automedicação e a idade dicotomizada $(p<0,05)$

\begin{tabular}{|l|c|c|c|c|}
\multirow{2}{*}{ Automedicação } & \multicolumn{2}{|c|}{$\begin{array}{c}\text { Teste } \\
\text { Idade }\end{array}$} & \multirow{2}{*}{$\begin{array}{c}\text { Total } \\
\text { (pearson }\end{array}$} & $\begin{array}{c}\text { (\%) A } \\
\text { qui-quadrado) }\end{array}$ \\
\cline { 1 - 4 } Sim & 72,2 & 27,8 & 100 & $\mathrm{P}=0,023$ \\
\hline Não & 45,7 & 54,3 & 100 & \\
\hline
\end{tabular}

Quem pratica automedicação, utiliza predominantemente doses subterapêuticas ou terapêuticas, enquanto que quem não recorre à automedicação utiliza geralmente as últimas (Quadro IV). O tempo esperado até iniciar automedicação é predominantemente maior que uma hora, enquanto a não automedicação tem início até à primeira hora, em mais de metade dos utentes (Quadro V).

Aplicando o teste do qui-quadrado e ao nível de significância de $5 \%$ não foram encontradas associações estatisticamente significativas $(p<0,05)$ entre a automedicação e as seguintes variáveis: raça, nível de escolaridade, medicação habitual realizada, localização da dor, duração do episódio doloroso e intensidade da dor.

\section{DISCUSSÃO}

$\mathrm{Na}$ amostra em estudo, a frequência relativa da dor aguda, consoante a sua localização, encontra semelhanças com estudos internacionais, ${ }^{10-12}$ exceptuando a maior frequência de mialgias e cefaleias relacionadas com a síndrome gripal. Esta discrepância está possivelmente relacionada com o facto de o inquérito ser relativo ao mês de Janeiro. Outras diferenças podem ser por esses estudos incluírem também localizações de dor crónica.

No presente estudo verificou-se que a terapêutica 
QUADRO IV. Análise da associação entre a automedicação e a dose farmacológica utilizada $(p<0,05)$

\begin{tabular}{|c|c|c|c|c|c|}
\hline \multirow[b]{2}{*}{ Automedicação } & \multicolumn{3}{|c|}{ Dose farmacológica utilizada } & \multirow{2}{*}{$\begin{array}{c}\text { Total } \\
(\%)\end{array}$} & \multirow{2}{*}{$\begin{array}{c}\text { Teste } \\
\text { (pearson qui quadrado) }\end{array}$} \\
\hline & subterapêutica & terapêutica & supraterapêutica & & \\
\hline Sim & 46,7 & 50,0 & 3,3 & 100 & \multirow{2}{*}{$p=0,011$} \\
\hline Não & 15,2 & 66,6 & 18,2 & 100 & \\
\hline
\end{tabular}

\begin{tabular}{|c|c|c|c|c|c|c|}
\hline \multirow[b]{2}{*}{ Automedicação } & \multicolumn{4}{|c|}{ Tempo antes de início da terapêutica } & \multirow{2}{*}{$\begin{array}{c}\text { Total } \\
(\%)\end{array}$} & \multirow{2}{*}{$\begin{array}{c}\text { Teste } \\
\text { (pearson qui quadrado) }\end{array}$} \\
\hline & de imediato & $\leq 1 \mathrm{~h}$ & $\leq 6 \mathrm{~h}$ & $>6 h$ & & \\
\hline Sim & 11,1 & 25,0 & 36,1 & 27,8 & 100 & \multirow{2}{*}{$p=0,021$} \\
\hline Não & 34,4 & 17,1 & 11,4 & 37,1 & 100 & \\
\hline
\end{tabular}

farmacológica foi mais utilizada do que a não farmacológica, sendo na sua maioria constituída por paracetamol, seguido pelos AINEs, resultados esses que são semelhantes aos obtidos por Hazard Vallerand. ${ }^{13}$ Também a associação entre o sexo e a dor, bem como entre o sexo e a automedicação, estão de acordo com os resultados do referido estudo, sugerindo-se uma futura investigação para compreensão das causas subjacentes a esta tendência.

A prevalência da automedicação com base em directrizes não qualificadas foi idêntica à com base em recomendações profissionais $(50,0 \%$ vs $44,5 \%$, respectivamente), o que é também referido por Montano Alonso. ${ }^{14}$ Estes autores determinaram também que a utilização de panfletos/posters informativos não atingia o objectivo de tornar a automedicação mais correcta, ao contrário da educação individual. Uma vez que uma parte importante da amostra referiu automedicar-se com base na informação fornecida pelo médico em situações dolorosas anteriores, é possível que o reforço da educação dada pelo médico a respeito do medicamento prescrito e das suas indicações terapêuticas aumente a correcção da automedicação neste grupo. Seria interessante elaborar um estudo neste sentido. Sugere-se que os utentes possam ser mais bem esclarecidos sobre as propriedades dos fármacos, na tentativa de diminuir o seu desconhecimento e a proporção de medicação por fundamentos não qualificados. ${ }^{14}$

Determinaram-se mais queixas dolorosas na faixa etária dos 26-35 anos e maior automedicação no grupo etário abaixo dos 45 anos. Não foram encontradas relações significativas entre a idade e a terapêutica efectuada, contrariamente aos achados de Vallerand $\mathrm{AH} .{ }^{13}$ Esta discordância relaciona-se possivelmente com diferenças entre os objectos de estudo: episódios de dor aguda autolimitada versus todas as queixas dolorosas. Pondera-se que, possivelmente, ao longo da vida aumentam as queixas dolorosas devidas a dor crónica e o seu impacto nas actividades diárias, diminuindo a frequência ou a valorização das dores agudas. Desse modo, os mais idosos podem considerar o tratamento das últimas menos importante que os indivíduos de escalões etários mais jovens. ${ }^{13}$ Pensa-se também que o tratamento da sua dor crónica poderá reduzir significativamente o número de episódios dolorosos agudos neste grupo.

No entanto, a prevalência de automedicação observada no grupo acima dos 45 anos não é menosprezável, e poderá ser importante dada a possível maior polimedicação neste escalão etário.

Não foram encontrados estudos disponíveis para corroborar a associação significativa entre a automedicação e um maior tempo de espera até ao início da terapêutica, entre a automedicação e doses subterapêuticas e a não automedicação com doses terapêuticas, que foram encontradas neste estudo.

Pondera-se que os utentes tenham maior segurança na prescrição pelo médico ou farmacêutico, pelo que 
iniciam mais rapidamente a terapêutica. Não foram apuradas razões para que a frequência de tomas acima da posologia recomendada ${ }^{9}$ tenha sido superior no grupo que não se automedicou. Não foi aqui encontrada uma associação significativa entre o prejuízo da dor nas actividades diárias e a estratégia terapêutica, o que nos leva a supor que o prejuízo causado pela dor não é critério para a opção pela automedicação ou pela consulta ao profissional de saúde.

É relevante que $88,8 \%$ dos doentes obtivesse remissão da dor. Se uma parte importante dos doentes utiliza doses subterapêuticas, esta eficácia poderá talvez dever-se a vários factores não estudados, entre os quais o efeito placebo ou a baixa intensidade da dor.

A respeito do grau de confiança nas variadas fontes de informação para a saúde, é positivo verificar a elevada confiança atribuída tanto aos médicos como aos farmacêuticos e enfermeiros, comparativamente às outras fontes.

O tipo de estudo efectuado é adequado aos objectivos pretendidos. O recurso à amostragem de conveniência é uma limitação do presente estudo. Considera-se que a selecção dos entrevistados mediante a sua presença no Centro de Saúde não é a mais correcta, uma vez que não é representativa de todos os utentes do mesmo nem aleatória, estando assim excluídos todos os utentes que não frequentaram o Centro de Saúde no período estudado. Nos dois dias em que foi realizada a colheita de dados, efectuaram-se entrevistas durante uma manhã e duas tardes. A exclusão de um período matinal diminuiu a representação na amostra dos utentes que frequentam o Centro de Saúde nesse horário, eventualmente com características diferentes dos frequentadores do período da tarde. A exclusão dos restantes dias da semana também pode ter incluído maior representação relativa de grupos particulares de utentes e deixado fora do estudo outros grupos.

Outras limitações do estudo são a utilização de escalas e classificações cuja validade não foi estudada previamente e a não realização de uma análise prévia do questionário criado, mediante teste-piloto.

A utilização de perguntas de resposta aberta deve-se à necessidade de conhecimentos da área da saúde para responder às questões. A opção pelo método de entrevista tornou possível a inclusão de pessoas com baixa escolaridade, que correspondem a $38,2 \%$ dos entrevis- tados ( $1^{\circ}$ ciclo ou menos), mas introduziu vieses relacionados com o entrevistador. Procurou-se a minimização dos mesmos, mediante clarificação dos entrevistadores a respeito dos objectivos de cada questão e treino de padronização, mas não se determinou a influência deste aspecto.

A realização do questionário em salas de espera do Centro de Saúde e a utilização de estudantes de medicina como entrevistadores introduzem um viés, relacionado com as atitudes socialmente esperadas. Porque não foi especificada a prevalência de analgésicos e anti-inflamatórios na medicação habitual realizada pelos indivíduos entrevistados, não foi possível o estudo comparativo da incidência de dor aguda em utentes que tomam habitualmente um fármaco destes grupos e os que não o fazem, e da frequência da sobreposição de efeitos farmacológicos idênticos na medicação habitual e na medicação para a dor aguda, o que poderia ter tido interesse. Não foi estudada a influência deste aspecto, mas presume-se que é possível que a prevalência da automedicação desinformada e confiança nas medicinas alternativas e populares se encontrem subestimadas, e o nível de confiança nos profissionais de saúde se encontre sobrestimado.

A consulta de um técnico de estatística reforça a fiabilidade dos resultados obtidos.

Sugere-se futuramente a realização de um estudo em que a dimensão da amostra necessária fosse determinada de forma a ser representativa e os entrevistados fossem seleccionados aleatoriamente através dos registos dos utentes na base de dados do Centro de Saúde. Desse modo, seria mais adequada a entrevista com recurso à chamada telefónica previamente notificada mediante aviso postal. A colheita dos dados deveria ser respectiva a um período de pelo menos seis meses ou realizada em pelo menos quatro meses correspondentes a diferentes estações do ano.

\section{CONCLUSÕES}

A gestão eficiente dos episódios de dor aguda é fundamental, pela frequência e impacto destes na saúde individual e da população. À luz dos actuais princípios éticos, é desejável que o doente assuma um papel de relevo na salvaguarda da sua saúde, partilhando responsabilidades com os profissionais. Desse modo, é essencial o fornecimento de informações adequadas à 
população em geral, para que os indivíduos desenvolvam instrumentos de decisão conscientes, eficazes e que minimizem a ocorrência de efeitos adversos. Este patamar ideal apenas poderá ser atingido através da realização de estudos que analisem as atitudes e crenças da população a respeito da sua saúde, os comportamentos que com base nestas desenvolvem e que procurem estudar a eficácia de diferentes métodos de transmissão de informação sobre saúde às populações.

O presente estudo revelou, de modo idêntico a outros estudos internacionais, uma elevada frequência da automedicação como estratégia terapêutica dos episódios álgicos, da qual uma fracção substancial se baseia na auto-decisão e em doses subterapêuticas, tendência esta mais marcada no sexo feminino e nos escalões etários mais jovens.

Considerando as limitações deste estudo, seria importante a realização de estudos aleatorizados, de grandes dimensões e de maior longitudinalidade, com o objectivo de caracterizar mais fielmente a população que se automedica e quais as informações/crenças que servem de base às suas tentativas terapêuticas, nomeadamente no que se relaciona com a escolha dos agentes terapêuticos e sua utilização. Considera-se também de grande relevância efectuar estudos que determinem o impacto da educação individual fornecida pelo Médico de Família na eficácia e segurança da automedicação de episódios álgicos agudos autolimitados, nomeadamente daqueles cuja frequência se revelou mais elevada (cefaleias, mialgias e raquialgias).

\section{REFERÊNCIAS BIBLIOGRÁFICAS}

1. International Association for the Study of Pain. Disponível em: http:// www.iasp-pain.org/ [acedido em 08/02/2007].

2. American Pain Foundation. Disponível em: http://www.painfoundation. org/ [acedido em 08/02/2007].

3. USA Today. Disponível em: http://www.usatoday.com/news/health/ [acedido em 08/02/2007].

4. Aljinović -Vuciić V, Trkulja V, Lackoviić Z. Content of home pharmacies and self-medication practices in households of pharmacy and medical students in Zagreb, Croatia: findings in 2001 with a reference to 1977. Croat Med J 2005 Feb; 46 (1): 74-80.
5. Portal do Governo, Governo da República Portuguesa. Disponível em: http://www.portugal.gov.pt/ [acedido em 07/02/2007].

6. Njah M, Ben Abdelaziz A, Naceur C, Yazid B, Nouira A, Ajmi T. Attitudes et pratiques de la population du Sahel Tunisien à l'égard de l'usage des médicaments. Tunis Med 2002 Mai; 80 (5): 249-54.

7. Thomas J, Straus WL, Bloom BS. Over-the-counter nonsteroidal antiinflammatory drugs and risk of gastrointestinal symptoms. Am J Gastroenterol 2002 Sep; 97 (9): 2215-9.

8. Wilcox CM, Cryer B, Triadafilopoulos G. Patterns of use and public perception of over-the-counter pain relievers: focus on nonsteroidal antiinflammatory drugs. J Rheumatol 2005 Nov; 32 (11): 2218-24.

9. Prontuário Terapêutico Online. Disponível em: http://www.infarmed. pt/prontuario/index.php [acedido em 08/02/2007].

10. Watkins EA, Wollan PC, Melton LJ 3rd, Yawn B. A population in pain: report from the Olmsted County health study. Pain Med 2008 Mar; 9 (2): 166-74.

11. Arrais PS, Coelho HL, Batista MC, Carvalho ML, Righi RE, Arnau JM. Perfil da automedicação no Brasil. Rev Saude Publica 1997 Fev; 31 (1): 71 $-7$.

12. Bassols A, Bosch F, Baños JE. How does the general population treat their pain? A Survey in Catalonia, Spain. J Pain Symptom Manage 2002 Apr; 23 (4): 318-28.

13. Vallerand AH, Fouladbakhsh J, Templin T. Patients' choices for the selftreatment of pain. Appl Nurs Res 2005 May; 18 (2): 90-6.

14. Montaño Alonso A, Torelló Iserte J, Castillo Ferrando JR, Cayuela Dominguez A, Moreno Gallego I, Fernández Díez P. Conocimientos y actitud de los usuários en relación al empleo de AINE: studio de intervención. Aten Primaria 1997 Jul-Ago; 20 (3): 114, 116-20.

\section{ENDEREÇO PARA CORRESPONDÊNCIA:}

Flávio Simões

Rua Henriques Nogueira, $n^{\circ} 21 \mathrm{r} / \mathrm{c}$

2560-340 Torres Vedras

Tlm: +351919534020

E-mail: peaceweapon@gmail.com

\section{AGRADECIMENTOS}

Agradece-se a contribuição do Centro de Saúde Cacém (extensão Olival), nas pessoas da Dra. Violeta Pimpão e Dra. Luísa Carvalho pelo auxílio prestado na formulação do estudo e autorização para a colheita dos dados. Agradece-se também ao Prof. Doutor Armando Brito de Sá pelo seu papel de consultor científico e revisor crítico do estudo.

Por fim, agradece-se o apoio do Laboratório de Biomatemática da FML, na pessoa da Dra. Teresa Rodrigues, pelo auxílio técnico prestado na análise e tratamento dos dados. 


\section{ABSTRACT}

Introduction: The treatment of pain is a capacity shared by the physician and the patients. Well-informed self-medication is beneficial for the population and for the Health System; however, frequent mistakes add to the risk of adverse effects.

Goals: To depict the self-limited episodes of acute pain and the respective therapeutic management featured in the examined sampling. To study the self-medication cases in terms of frequency and connection with socio-demographic factors.

Methods: The cross-sectional study was based on a sampling of 352 patients from the Cacém Health Care Centre (Olival extension), using one questionnaire for each of the personal interviews concerning self-limited acute pain and its therapeutic management during the 30 preceding days.

Results: There were 89 episodes of self-limited acute pain (25.3\%), mainly women. The most frequent episodes consisted of headaches (48.3\%), myalgia and flu syndrome-related headaches (10.1\%) and rachialgia (7.9\%). Of the 89 patients that referred an episode of self-limited acute pain in the 30 days prior to the questionnaire, $63.0 \%$ relied solely on pharmacotherapy, while $12.4 \%$ resorted to non-pharmacological therapeutic as well. The most widely used drugs were Paracatemol (50.7\%) and NSAIDs (31.3\%). Self-medication was frequent (46.5\%), mostly on women of less than 45 years of age; it was associated with sub-therapeutic drugs and was based on prior medical indication or self-decision.

Discussion: It was observed that there are significant associations between self-medication and a few socio-demographic factors and that self-medication hinders an adequate posology. The importance of self-decision in the context of self-medication was also noted; the study suggests that the patients must be better clarified about the properties of the drugs.

Keywords: Pain epidemiology; Frequency; Acute pain; Self-medication. 


\section{ANEXO \\ ANÁLISE DAS TERAPÊUTICAS REALIZADAS EM EPISÓDIOS DE DOR AGUDA, NOS 30 DIAS ANTERIORES}

1) Teve dor que se iniciou nos últimos 30 dias?

Sim $\square$ Não $\square$

Se Sim:

Crónica/agudização de dor crónica

Episódio agudo não resolvido

Episódio agudo resolvido

2) Idade

3) Sexo $F \quad \square$

4) Raça

5) Escolaridade

Iletrados

$\begin{array}{ll}1^{\circ} \text { ciclo } / 4^{\mathrm{a}} \text { classe } & 12^{\mathrm{o}} \text { ano } \\ 2^{\mathrm{o}} \text { ciclo } / 6^{\mathrm{o}} \text { ano } & \text { Curso profissional }\end{array}$

$3^{\circ}$ ciclo $/ 9^{\circ}$ ano Ensino Superior

6) Medicação habitual

\section{Em relação ao(s) episódio(s) de dor aguda}

7) Dor

Número de episódios:

Tipo/ Localização da dor:

Intensidade

Sem prejuízo nas actividades do dia-a-dia

Com prejuízo ligeiro nas actividades do dia-a-dia

Com prejuízo moderado nas actividades do dia-a-dia

Com prejuízo grave nas actividades do dia-a-dia

Duração:

8) Terapêutica não farmacológica?

Sim $\square$ Não $\square$

Se Sim qual?
9) Terapêutica farmacológica?

Nome:

Dose/ $\mathrm{n}^{\circ}$ de comprimidos:

10) Quanto tempo após o início da dor iniciou a medicação?

11) Quem recomendou o(s) fármaco(s)?

Médico

Farmacêutico/Enfermeiro

Conhecido(s)

O Próprio

12) Se automedicação, em que fundamento se baseia?

Recomendação médica anterior

Auto-decisão

Outras. Quais?

13) Quanto tempo durou a terapêutica?

14) Remissão da sintomatologia dolorosa?

Sim $\square$ Não

Se Não o que fez?

Se foi ao médico qual a terapêutica prescrita?

15) Grau de confiança:

Médico

1 - Nenhuma

Farmacêutico/Enfermeiro

2 - Pouca

Medicinas Alternativas

3 - Indiferente

Sabedoria Popular

4 - Boa

Comunicação social

Data: 1

Obrigado pela sua colaboração. 